\title{
Emerging Value: The Chick Chorioallantoic Membrane (CAM) Model in Oral Carcinogenesis Research
}

\author{
Scanlon CS, Inglehart RC and D'Silva $\mathrm{NJ}^{*}$
}

Department of Periodontics and Oral Medicine, School of Dentistry, University of Michigan, Ann Arbor, MI, USA

\begin{abstract}
New questions and technological advances in cancer research have led to renewed relevance for the chicken Chorioallantoic Membrane (CAM) model. The CAM model is a powerful tool to study early events of tumor progression of head and neck cancer. Several advantages of the CAM model over other in vivo models include its clinical relevance, cost effectiveness and the wide accessibility of chicken eggs. These benefits make the CAM model of cancer progression an attractive in vivo option for global oral cancer researchers.
\end{abstract}

Keywords: Invasion; Tumor progression; In vivo models; Chicken embryo

The embryonic chicken is among the most well-characterized and useful in vivo biological systems [1]. The chorioallantoic membrane (CAM) assay is an emerging model of tumor progression using the embryonic chicken. The CAM is a very vascular structure that surrounds the chick embryo. It allows for exchange of dissolved oxygen and carbondioxde, similar to the function of the placenta of a developing mammal. The CAM is comprised of an upper chorionic epithelium, intervening mesenchyme, and lower allantoic epithelium. The chorionic epithelium is structurally similar to human epithelium and has a collagen-IV - rich basement membrane. In the CAM assay, a small opening is made in the shell of a fertilized egg, allowing a tumor graft to be seeded directly on the chorionic epithelium. Grafted tumor cells invade through the basement membrane of the chorionic epithelium and into vascular structures in the underlying mesenchyme, thereby metastasizing to distant structures and organs including the liver and lungs of the developing chicken and the lower CAM [2].

The first tumor grafts on the CAM were successfully performed in 1913, establishing that the CAM readily accepts xenografts [2]. This discovery led to the development of multiple carcinogenesis assays using the CAM, which have become prominent methods to assess tumor angiogenesis, progression and metastasis [2,3]. Although the basic technique of grafting tumors to the CAM has changed very little, powerful technological advances allow scientists to use the CAM for assays of increasing complexity. These advances have led to renewed attention to this established model, particularly in the past decade (Figure 1). Since 2010, nearly one hundred cancer-related publications using the CAM model are listed on PubMed annually in peer-reviewed journals.

The CAM is used to study a wide range of cancers, including breast and prostate cancers. Our laboratory recently reported the use of the CAM model to simultaneously study multiple aspects of Head and Neck Squamous Cell Carcinoma (HNSCC) tumor progression, including tumor growth, invasion, metastasis and angiogenesis [2]. In this model, a small window is made through the shell of a fertilized egg early in development to expose the CAM. Tumor cells are seeded on the CAM of the developing chicken to provide an in vivo model of HNSCC progression. Within two to seven day of seeding, several parameters of HNSCC progression can be assessed. For example, invasion of the basement membrane is observable within two days of seeding whereas metastasis is investigated at days 5-7 after seeding of HNSCC cells on the CAM. This is similar to the sequence of clinical progression of HNSCC. For example, invasion of the basement membrane is required for transformation of precancerous lesions into HNSCC [4].

The CAM model has also been used to study epithelial dysplasia (pre-cancer) and peri-tumor lymphatic vessel density, as well as to test

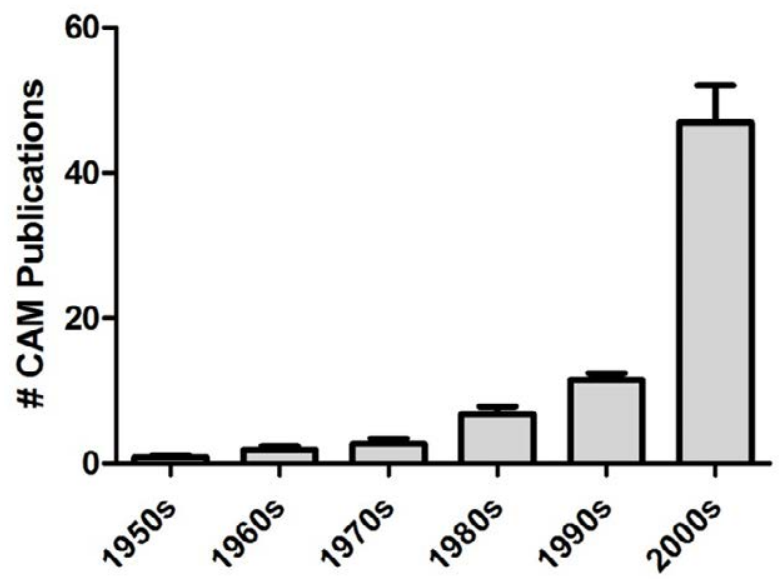

Figure 1: The CAM emerges as a standard model of carcinogenesis. "Chorioallantoic membrane" and "cancer" were entered as search terms on PubMed (http://www.ncbi.nlm.nih.gov/pubmed/), and the results were sorted by date of addition to PubMed database. The number of articles published per year was quantified, and Student's t-test was used to analyze the difference in the number of publications using the CAM method between decades. Between the 1970 s and 1980s and beyond there is a statistically significant increase in the number of articles, and the largest increase occurred between the 1990s and 2000s. Given this trend, it is likely that an increasing number of cancer researchers will employ the CAM model.

*Corresponding author: Nisha J. D'Silva, 1011 N. University Ave, Rm 5217, Dept of Periodontics and Oral Medicine, University of Michigan School of Dentistry, Ann Arbor, MI 48109-1078, USA, Tel: (734) 764-1543; Fax: (734) 764-2469; E-mail: njdsilva@umich.edu

Received August 12, 2013; Accepted August 20, 2013; Published August 26 2013

Citation: Scanlon CS, Inglehart RC, D'Silva NJ (2013) Emerging Value: The Chick Chorioallantoic Membrane (Cam) Model in Oral Carcinogenesis Research. J Carcinogene Mutagene S5: 005. doi:10.4172/2157-2518.S5-005

Copyright: @ 2013 Scanlon CS, et al. This is an open-access article distributed under the terms of the Creative Commons Attribution License, which permits unrestricted use, distribution, and reproduction in any medium, provided the original author and source are credited. 


\begin{tabular}{|c|c|}
\hline HNSCC Cancer Phenotype Studied & Reference(s) \\
\hline Angiogenesis & {$[2,5-13]$} \\
\hline Biomarker Expression & {$[2,6,7]$} \\
\hline Drug Testing & {$[6-9,12-15]$} \\
\hline Dysplasia Study & {$[16]$} \\
\hline Invasion & {$[2,6,10,17-19]$} \\
\hline Lymphatic Vessel Density & {$[20]$} \\
\hline Metastasis & {$[13-15,17,21]$} \\
\hline Tumor Growth & {$[2,6,7,10,22]$} \\
\hline
\end{tabular}

Table 1: The use of the CAM model in HNSCC research. The terms "chorioallantoic" and "head and neck cancer" were used to identify articles listed on Pubmed that use the CAM model to study HNSCC. The articles from the resulting search are organized according to the phenotype investigated using the CAM model system.

drugs for HNSCC. The study and related phenotypes are summarized in Table 1.

The CAM model of HNSCC has several benefits over murine in vivo models. The CAM closely replicates the tissue complexity of oral mucosa and therefore is an excellent model for HNSCC progression. Most murine models of HNSCC require subcutaneous or submucosal injection of cancer cells. Because the injected cells artificially bypass the basement membrane, injection-based murine models cannot replicate destruction of the basement membrane with subsequent invasion. This important phenotype is an essential step for transformation of a premalignant lesion into HNSCC [4]. However, in the CAM model of HNSCC, tumor cells must degrade the basement membrane of the chorionic epithelium to invade, closely replicating HNSCC development. Given that some recent work emphasizes the impact on invasion of structures and channels found in true extracellular matrix, it is attractive to study invasion in vivo [23,24].

Many technological advances are giving a fresh perspective on the value of CAM tumors. It is now possible to use stop-motion videomicroscopy to continuously monitor a target's activity over a period of days [25]. Unlike traditional murine models, the CAM model is able to exploit the promise of these new techniques. While both murine and CAM models provide in vivo results, tumors on the CAM are more readily observed and quantified. For similar reasons, the CAM model also offers easier study of angiogenesis.

Another advantage of the CAM model is the short duration (maximum 1 week) required to assess even late events in tumor progression, such as metastasis. This may take several weeks to months to assess in murine models [27]. This long duration increases the cost and time required for investigations. The rapid turnaround of results in the CAM model allows transient transfection studies, which cannot be performed in murine models. For example, the impact of an siRNA on downregulation of a protein lasts only a few days. This timeframe is insufficient for changes in tumor growth to be investigated in mice, which take several days to develop tumors.

Due to the lack of an immune system in the early chicken embryo, the CAM system readily accepts many types of xenografts. Very small numbers of cells are needed for xenograft experiments, and metastasis can be quantified very accurately through PCR-based methods [27]. The cost of mice limits the number of mammals that can be used for each experiment. The CAM model provides an opportunity to perform large in vivo experiments at a fraction of the cost of murine-based models of HNSCC. The cost effectiveness and easy accessibility of the CAM system enhance the appeal of this in vivo model, particularly when funding resources are limited.
The CAM model of tumor progression has some limitations. Perhaps the most significant limitation is that the mouse is currently accepted as the gold standard for in vivo biological studies. An initial investment of time and materials is necessary to set up the CAM protocol, which requires technical dexterity. This is also true of some murine HNSCC models such as the tongue and floor-of-mouth models [28].

The chick embryo becomes fully immunocompetent by day 18 [26]. The developing immune system of the embryonic chicken limits the duration of the study. Additionally, the lack of an immune system prevents the investigation of tumor-host immune system interactions. However, investigations of human HNSCC in mouse also require the use of immunodeficient mice. The larger sample sizes used for CAM experiments yield a large amount of data from multiple phenotypes requiring extensive time for analysis.

Overall, the scientific benefits of the CAM model make it an attractive option to all cancer biologists. The practical advantages of the CAM model over rodent models make it an accessible option to scientists in developing institutions. For these reasons, and because the CAM brings the study of these processes into a uniform setting where interactions can be examined, the popularity of the CAM model will continue to increase.

\section{Acknowledgement}

This work was supported by NIDCR DE16920-01 and DE018512- 01 (NJD), DE021293 (CSS) and the Elizabeth Caroline Crosby Fund

\section{References}

1. Stern CD (2005) The chick; a great model system becomes even greater. Dev Cell 8: 9-17.

2. Liu M, Scanlon CS, Banerjee R, Russo N, Inglehart RC, et al. (2013) The Histone Methyltransferase EZH2 Mediates Tumor Progression on the Chick Chorioallantoic Membrane Assay, a Novel Model of Head and Neck Squamous Cell Carcinoma. Transl Oncol 6: 273-281.

3. Murphy JB (1913) Transplantability Of Tissues To The Embryo Of Foreign Species : Its Bearing On Questions Of Tissue Specificity And Tumor Immunity. J Exp Med 17: 482-493.

4. Auerbach R, Arensman R, Kubai L, Folkman J (1975) Tumor-induced angiogenesis: lack of inhibition by irradiation. Int J Cancer 15: 241-245.

5. Ossowski L, Reich E (1980) Experimental model for quantitative study of metastasis. Cancer Res 40: 2300-2309.

6. Scanlon CS, Van Tubergen EA, Inglehart RC, D'Silva NJ (2013) Biomarkers of epithelial-mesenchymal transition in squamous cell carcinoma. J Dent Res 92: 114-121.

7. Hseu YC, Wu CR, Chang HW, Kumar KJ, Lin MK, et al. (2011) Inhibitory effects of Physalis angulata on tumor metastasis and angiogenesis. $\mathrm{J}$ Ethnopharmaco 135: 762-771.

8. Kim SA, Kwon SM, Kim JA, Kang KW, Yoon JH, et al. (2011) 5'-Nitroindirubinoxime, an indirubin derivative, suppresses metastatic ability of human head and neck cancer cells through the inhibition of Integrin I21/FAK/Akt signaling. Cancer Lett 306: 197-204.

9. Nagasawa H, Mikamo N, Nakajima $Y$, Matsumoto $H$, Uto $Y$, et al. (2003) Antiangiogenic hypoxic cytotoxin TX-402 inhibits hypoxia-inducible factor 1 signaling pathway. Anticancer Res 23: 4427-4434.

10. Oh SH, Kim WY, Kim JH, Younes MN, El-Naggar AK, et al. (2006) Identification of insulin-like growth factor binding protein-3 as a farnesyl transferase inhibitor $\mathrm{SCH} 66336$-induced negative regulator of angiogenesis in head and neck squamous cell carcinoma. Clin Cancer Res 12: 653-661.

11. Petruzzelli GJ, Snyderman CH, Johnson JT, Myers EN (1993) Angiogenesis induced by head and neck squamous cell carcinoma xenografts in the chick embryo chorioallantoic membrane model. Ann Otol Rhinol Laryngol 102: 215 221. 
Citation: Scanlon CS, Inglehart RC, D'Silva NJ (2013) Emerging Value: The Chick Chorioallantoic Membrane (CAM) Model in Oral Carcinogenesis Research. J Carcinogene Mutagene S5: 005. doi:10.4172/2157-2518.S5-005

12. Pisanti S, Borselli C, Oliviero O, Laezza C, Gazzerro P, et al. (2007) Antiangiogenic activity of the endocannabinoid anandamide: correlation to its tumor-suppressor efficacy. J Cell Physiol 211: 495-503.

13. Gronau S, Thess B, Riechelmann H, Fischer $Y$, Schmitt A, et al. (2006) An autologous system for culturing head and neck squamous cell carcinomas for the assessment of cellular therapies on the chorioallantois membrane. Eur Arch Otorhinolaryngol 263: 308-312.

14. Nyberg P, Moilanen M, Paju A, Sarin A, Stenman UH, et al. (2002) MMP9 activation by tumor trypsin- 2 enhances in vivo invasion of human tongue carcinoma cells. J Dent Res 81: 831-835.

15. Yang SF, Yang WE, Kuo WH, Chang HR, Chu SC, et al. (2008) Antimetastatic potentials of flavones on oral cancer cell via an inhibition of matrix-degrading proteases. Arch Oral Biol 53: 287-294.

16. Hoppenheit C, Hüttenberger D, Foth HJ, Spitzer WJ, Reichert TE, et al. (2006) Pharmacokinetics of the photosensitizers aminolevulinic acid and aminolevulinic acid hexylester in oro-facial tumors embedded in the chorioallantois membrane of a hen's egg. Cancer Biother Radiopharm 21: 569-578.

17. Chang HL, Pieretti-Vanmarcke R, Nicolaou F, Li X, Wei X, et al. (2011) Mullerian inhibiting substance inhibits invasion and migration of epithelial cancer cell lines. Gynecol Oncol 120: 128-134.

18. Easty DM, Easty GC, Baici A, Carter RL, Cederholm-Williams SA, et al. (1986) Biological studies of ten human squamous carcinoma cell lines: an overview. Eur J Cancer Clin Oncol 22: 617-634.

19. Van Tubergen EA, Banerjee R, Liu M, Vander Broek R, Light E, et al. (2013) Inactivation or loss of TTP promotes invasion in head and neck cancer via transcript stabilization and secretion of MMP9, MMP2, and IL-6. Clin Cancer Res 19: 1169-1179.
20. Huang HZ, Tang HK (2005) [Interaction of vascular endothelial growth factor-C over-expression with tongue squamous cell carcinoma cell line Tca8113 with peri-carcinoma lymphatics]. Zhonghua Kou Qiang Yi Xue Za Zhi 40: 126-128.

21. Teruszkin Balassiano I, Alves De Paulo S, Henriques Silva N, Curié Cabral M, da Gloria da Costa Carvalho M (2001) Metastatic potential of MDA435 and Hep2 cell lines in chorioallantoic membrane (CAM) model. Oncol Rep 8: 431 433.

22. Bragado P, Estrada Y, Sosa MS, Avivar-Valderas A, Cannan D, et al. (2012) Analysis of marker-defined HNSCC subpopulations reveals a dynamic regulation of tumor initiating properties. PLoS One 7: e29974.

23. Even-Ram S, Yamada KM (2005) Cell migration in 3D matrix. Curr Opin Cell Biol 17: 524-532

24. Weigelt B, Bissell MJ (2008) Unraveling the microenvironmental influences on the normal mammary gland and breast cancer. Semin Cancer Biol 18: 311-321.

25. Gligorijevic B, Condeelis J (2009) Stretching the timescale of intravital imaging in tumors. Cell Adh Migr 3: 313-315.

26. Ribatti D (2010) The chick embryo chorioallantoic membrane in the study of angiogenesis and metastasis. London: Springer.

27. Zijlstra A, Mellor R, Panzarella G, Aimes RT, Hooper JD, et al. (2002) A quantitative analysis of rate-limiting steps in the metastatic cascade using human-specific real-time polymerase chain reaction. Cancer Res 62: 70837092.

28. Henson B, Li F, Coatney DD, Carey TE, Mitra RS, et al. (2007) An orthotopic floor-of-mouth model for locoregional growth and spread of human squamous cell carcinoma. J Oral Pathol Med 36: 363-370.
This article was originally published in a special issue, Oncogenomics of the Head and Neck handled by Editor(s). Dr. Rui Amaral Mendes, Portuguese Catholic University, Portugal 